\title{
Look back, looking forward
}

\section{Understanding of anthropogenic climate change has evolved since the IPCC's First Assessment Report. Further progress relies on continued collaboration between observationalists and modellers.}

Over the course of the IPCC's history - from the First Assessment Report (FAR) in 1990 to the Fifth Assessment Report (AR5) in 2014 - considerable progress has been made in our understanding of anthropogenic climate change. Key advances can partially be attributed to improvements in modelling capabilities, including resolution, addition of new or updated processes, and ultimately, computing power. However, model developments are inextricably linked to a simultaneous increase in observational analyses and corresponding expansion of the observational network. Records are now arguably of sufficient length to assess the skill of early projections, and further track advances in understanding over the past 27 years of the IPCC.

Several studies have illustrated that, despite the relative simplicity of early climate models, large-scale temperature predictions from the FAR have been surprisingly consistent with observed changes, both in terms of the magnitude and spatial pattern of anthropogenic warming ${ }^{1,2}$. While an impressive feat, our understanding of other variables has seen a much greater evolution from the first to the fifth AR, some examples of which are illustrated in this issue. Ice-sheet models, for instance, now have the capacity to explicitly simulate dynamical imbalances, and as a result, projections of sea-level rise contributions are now in close agreement with observations (page 672). Similarly, it was not until AR5 that models had improved to the extent that overall cloud feedbacks were first quantified as being positive within the IPCC's uncertainty framework (page 674). Developments in methane sources and sinks (page 678) and quantification of agricultural yield impacts (page 680) offer further evidence of advanced understanding.

While highlighting a few instances of early successes and continued developments, the wealth of climate change literature clearly emphasises the progress made since the FAR. However, we cannot be complacent. Building on the examples above, key glaciological processes remain unquantified, individual cloud feedback terms continue to be highly uncertain, apportionment of methane sources and sinks challenging, and agricultural assessments focussed on crops, omitting impacts on other produce relevant to food security. As acknowledged by all these authors, more and continued observations are required to drive progress, both in terms of improved model validation and process understanding. In an Interview, Veronika Eyring, chair of the Coupled Model Intercomparison Project Phase 6 (CMIP6), further stresses the importance of increased collaboration between observationalists and modellers (page 684).

These are exciting times for climate science. As CMIP6 gets underway (page 684) in preparation for the IPCC's Sixth Assessment Report, a wealth of new model data will soon be available, further enhancing our understanding of the physics, impacts, and mitigation potential of anthropogenic climate change. However, continued progress relies on enhanced collaboration between various user groups and communities.

References

1. Frame, D. J. \& Stone, D. A. Nat. Clim. Change 3, 357-359 (2013).

2. Stouffer, R. J. \& Manabe, S. Nat. Clim. Change 7, 163-165 (2017).

\section{Storms ahead}

\section{As the climate changes, extreme storm and flood events are increasing in intensity and frequency, exposing more people to their impacts. Resilience planning needs to start now to limit these impacts.}

Flooding and storms account for almost three-quarters of weather-related disasters. This past month has seen flooding in Asia and a series of powerful hurricanes in the Atlantic. Extreme events such as these are not caused by climate change - they have occurred throughout history - but previous benchmarks are no longer valid; 1-in-100-year events are happening more often, and those timescales are shortening.

Across India, Bangladesh and Nepal over 40 million people were affected by flooding in late August and early September. Heavy monsoon rains, the worst in 40 years, arrived with little warning and later than the normal peak of the monsoon season. A third of Bangladesh was underwater during the flooding, with around 8 million people affected, whereas India and Nepal had over 30 million and 1.7 million people affected, respectively. Cropland and housing was destroyed, meaning many have lost not only their homes but their livelihoods too. Over 1,200 people died across the region and the risk continues when the waters recede, with increases in mosquito-borne and waterborne diseases as well as food shortages.

Hurricane Harvey caused heavy rainfall in Houston, Texas, where urban expansion and impervious surfaces enhanced flooding. This was the third 1-in-500-year flood that the city has experienced since 1979 . The US National Weather service had to add a new colour to their precipitation scale to represent the rainfall from Harvey. The standard of floodplain design needs to be updated to handle events such as these.

Hurricane Irma, a Category 5 storm, raged across the Caribbean and on to Florida. It was the most powerful storm ever recorded in the Atlantic, with the warmer sea surface fuelling the hurricane. Irma was so strong that it was detected by earthquake sensors and maintained winds over 185 miles per hour for 37 hours. Whilst Harvey caused damage through heavy rainfall and associated flooding, Irma brought rainfall accompanied by strong winds and storm surge, changing the coastline of the region. The damage bill is expected to exceed US $\$ 100$ billion for each of these two hurricanes, and the hurricane season is not yet over.

The recovery efforts from such events can be a time to prepare for the changes ahead, with a shift in thinking needed for resilience planning. The built environment and major infrastructure projects need to consider not only what is needed in the future, but also the interaction with infrastructure that is already in place. The costs of mitigation can be large, but earlier investment can greatly limit both climate change and related expenses from extreme events. 